\title{
Shadowing Effects on Particle and Transverse Energy Production*
}

\author{
V. Emel'yanov ${ }^{\mathrm{a}}$, A. Khodinov ${ }^{\mathrm{a}}, \mathrm{S}$. R. Klein ${ }^{\mathrm{b}}$ and R. Vogt ${ }^{\mathrm{b}, \mathrm{c}}$ \\ ${ }^{a}$ Moscow State Engineering Physics Institute (Technical University), Kashirskoe Ave. \\ 31, Moscow, 115409, Russia \\ bLawrence Berkeley National Laboratory, Berkeley, CA 94720, USA \\ ${ }^{\mathrm{c}}$ Physics Department, University of California, Davis, CA 95616, USA
}

The effect of shadowing on the early state of ultrarelativistic heavy ion collisions and transverse energy production is discussed. Results are presented for $\mathrm{RHIC} \mathrm{Au}+\mathrm{Au}$ collisions at $\sqrt{s_{N N}}=200 \mathrm{GeV}$ and LHC Pb+Pb collisions at $\sqrt{s_{N N}}=5.5 \mathrm{TeV}$.

The proton and neutron structure functions are modified in the nuclear environment [1], referred to here as shadowing. In most shadowing models, such as gluon recombination, the structure function modifications should be correlated with the local nuclear density. The E745 experiment studied the spatial distribution of structure functions with $\nu N$ interactions in emulsion [2]. They found evidence of a spatial dependence but could not determine the form. The spatial dependence of shadowing is reflected in particle production as a function of impact parameter, $b$, which may be inferred from the total transverse energy, $E_{T}$, produced in a heavy ion collision [3].

At RHIC and LHC perturbative QCD processes are expected to be an important component of the total particle production. In particular, at early times, $\tau_{i} \sim 1 / p_{T} \leq 1 / p_{0} \sim 0.1$ fm for $p_{0} \sim 2 \mathrm{GeV}$, semihard production of low $p_{T}$ minijets sets the stage for further evolution [1].

The average transverse energy of minijet production is proportional to the initial energy density and is the hard scattering contribution to the average total transverse energy. It is calculated within a specific detector acceptance $\epsilon(y)$. At leading order, the average transverse energy for a given minijet flavor, $f$, as a function of impact parameter is

$$
\begin{aligned}
\bar{E}_{T}^{f}(b)=\frac{d \sigma^{f}\left\langle E_{T}^{f}\right\rangle}{d^{2} b}= & K_{\text {jet }} \int d^{2} r d p_{T}^{2} d y_{2} d y d z d z^{\prime} \sum_{\substack{i j=\\
\langle k l\rangle}} x_{1} F_{i}^{A}\left(x_{1}, p_{T}^{2}, \vec{r}, z\right) x_{2} F_{j}^{B}\left(x_{2}, p_{T}^{2}, \vec{b}-\vec{r}, z^{\prime}\right) \\
& \times \frac{\epsilon(y) p_{T}}{1+\delta_{k l}}\left[\delta_{f k} \frac{d \hat{\sigma}^{i j \rightarrow k l}}{d \hat{t}}(\hat{t}, \hat{u})+\delta_{f l} \frac{d \hat{\sigma}^{i j \rightarrow k l}}{d \hat{t}}(\hat{u}, \hat{t})\right]
\end{aligned}
$$

Identical particles in the final state are accounted for by the factor $1 /\left(1+\delta_{k l}\right)$. The ratio of the NLO to LO jet cross sections, $K_{\text {jet }}$, indicates the size of the NLO corrections. A

${ }^{*}$ This work was supported in part by the Director, Office of Energy Research, Division of Nuclear Physics of the Office of High Energy and Nuclear Physics of the U. S. Department of Energy under Contract No. DE-AC03-76SF0098. 
conservative $K_{\text {jet }}=1$ [5] gives a lower limit on minijet production. The cutoff $p_{0}$ is the lowest $p_{T}$ scale at which perturbative QCD is valid.

The nuclear parton densities, $F_{i}^{A}$, are assumed be the product of the nucleon density in the nucleus $\rho_{A}(\vec{r}, z)$, the nucleon parton density $f_{i}^{N}\left(x, Q^{2}\right)$, and a shadowing function $S^{i}\left(A, x, Q^{2}, \vec{r}, z\right)$ where $A$ is the atomic mass number, $x$ is the parton momentum fraction, $Q^{2}$ is the interaction scale, and $\vec{r}$ and $z$ are the transverse and longitudinal location of the parton so that $F_{i}^{A}\left(x, Q^{2}, \vec{r}, z\right)=\rho_{A}(\vec{r}, z) S^{i}\left(A, x, Q^{2}, \vec{r}, z\right) f_{i}^{N}\left(x, Q^{2}\right)$. In the absence of nuclear modifications, $S^{i}\left(A, x, Q^{2}, \vec{r}, z\right) \equiv 1$. If the nuclear structure functions are homogeneous, then the spatial effects factorize and $\bar{E}_{T}^{f}\left(b, p_{0}\right)$ is proportional to the number of nucleon-nucleon collisions, $T_{A B}(b)$. We have calculated our results with GRV 94 LO [6] and MRST LO [7] parton distributions. The GRV low $x$ gluon density is larger than the MRST and produces strikingly different results at LHC while the RHIC results are rather similar. We use three different parameterizations of shadowing, all based on nuclear deep inelastic scattering. The first parameterization, $S_{1}(A, x)$, treats quarks, antiquarks, and gluons identically without $Q^{2}$ evolution [8]. The others evolve with $Q^{2}$ and conserve baryon number and total momentum. The second, $S_{2}^{i}\left(A, x, Q^{2}\right)$, modifies the valence quarks, sea quarks and gluons separately and includes $Q^{2}$ evolution for $Q_{0}=2<Q<10$ $\mathrm{GeV}$ [9]. The third, $S_{3}^{i}\left(A, x, Q^{2}\right)$, evolves each parton type separately above $Q_{0}=1.5 \mathrm{GeV}$ [10]. We have studied the spatial dependence of shadowing using two parameterizations. If shadowing is proportional to the local density [3, 11,

$$
S_{\mathrm{WS}}^{i}=S^{i}\left(A, x, Q^{2}, \vec{r}, z\right)=1+N_{\mathrm{WS}}\left[S^{i}\left(A, x, Q^{2}\right)-1\right] \frac{\rho_{A}(\vec{r}, z)}{\rho_{0}},
$$

where $N_{\mathrm{WS}}$ is chosen so that $(1 / A) \int d^{2} r d z \rho_{A}(\vec{r}, z) S_{\mathrm{WS}}^{i}=S^{i}$. If instead, shadowing arises due to multiple interactions of the incident parton, shadowing should be proportional to the path length of the parton through the nucleus,

$$
S_{\mathrm{R}}^{i}\left(A, x, Q^{2}, \vec{r}, z\right)=\left\{\begin{array}{ll}
1+N_{\mathrm{R}}\left(S^{i}\left(A, x, Q^{2}\right)-1\right) \sqrt{1-\left(r / R_{A}\right)^{2}} & r \leq R_{A} \\
1 & r>R_{A}
\end{array} .\right.
$$

The normalization, $N_{\mathrm{R}}$, obtained after averaging over $\rho_{A}(\vec{r}, z)$, is slightly larger than $N_{\mathrm{Ws}}$. In both cases, at distances much greater than $R_{A}$, the nucleons behave as free particles while in the center of the nucleus, the modifications are larger than the average value $S^{i}$. We have also studied inhomogeneous shadowing assuming that shadowing is proportional to the path length through the nucleus and find that the central shadowing is somewhat stronger than with $S_{\mathrm{WS}}^{i}$ in Eq. (2).

The effect of the inhomogeneous shadowing, $S_{\mathrm{WS}}^{i}$ in Eq. (2), on $\bar{E}_{T}^{f}(b)$, calculated with the GRV 94 LO parton densities, is shown in Fig. 1 for CMS and STAR. The CMS and ALICE ratios are similar as are the STAR and PHENIX results. When $x$ lies in the shadowing region, central collisions are more shadowed than the average. In the antishadowing region, central collisions are more antishadowed than the average. When $b \sim R_{A}$, the homogeneous and inhomogeneous shadowing are approximately equal. When $b \sim 2 R_{A}$, shadowing/antishadowing is significantly reduced. As $b$ increases further, $S=1$ is approached asymptotically. When inhomogeneous shadowing is assumed to be proportional to $S_{\mathrm{R}}^{i}$, Eq. (3), we find stronger shadowing at low impact parameter and a somewhat faster approach to $S=1$ at large $b$. 

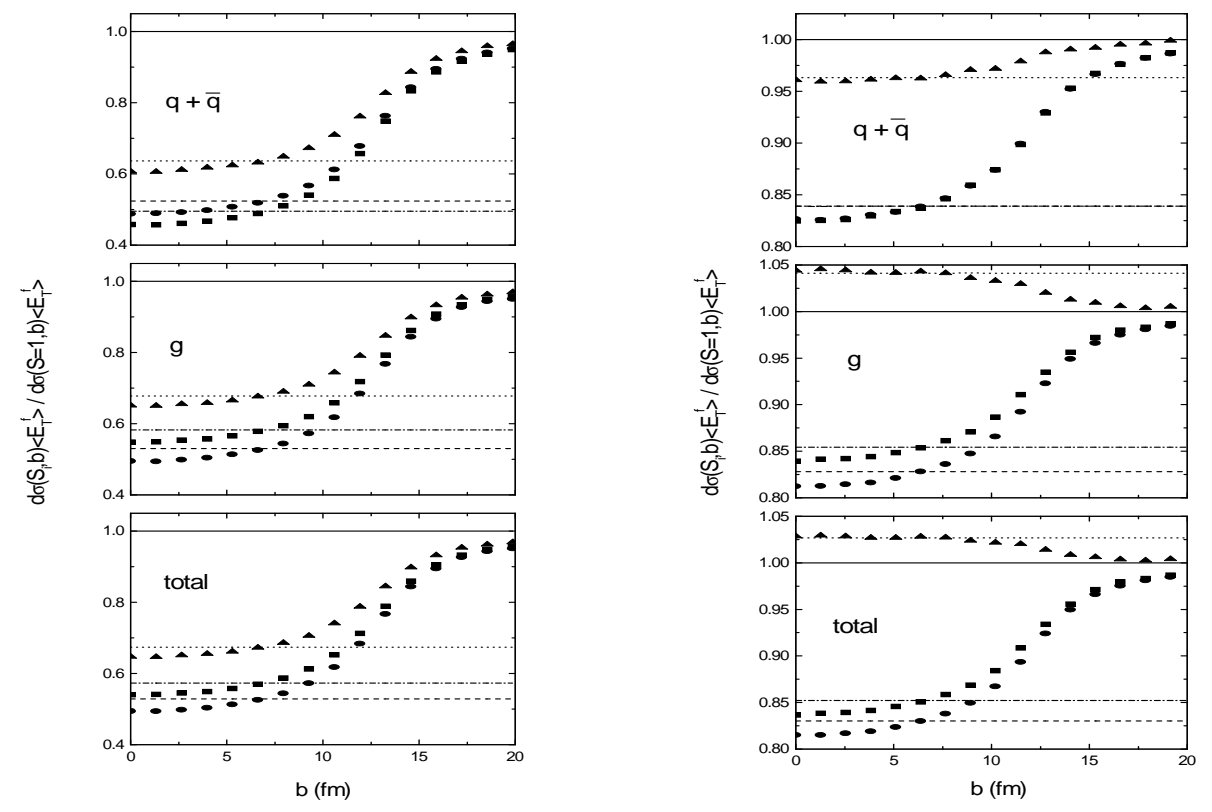

Figure 1. The impact parameter dependence of the first $E_{T}$ moment, compared to that with $S=1$ in CMS, $\left|y_{\max }\right| \leq 2.4$, at the LHC (left) and in STAR, $\left|y_{\max }\right| \leq 0.9$, at RHIC (right) calculated with the GRV $94 \mathrm{LO}$ distributions and $p_{0}=2 \mathrm{GeV}$. The upper plot shows the ratio for quarks and antiquarks, the middle plot is the gluon ratio and the lower plot is for the total. The horizontal lines show the homogeneous shadowing results: dashed for $S_{1}$, dot-dashed for $S_{2}$, and dotted for $S_{3}$. The inhomogeneous shadowing results using $S_{\mathrm{WS}}^{i}$ for $S_{1}$, circles, $S_{2}$, squares, and $S_{3}$, triangles are also shown [12].

We assume that the transverse energy distribution, $d \sigma / d E_{T}$, can be approximated by the Gaussian [5]

$$
\frac{d \sigma}{d E_{T}}=\int \frac{d^{2} b}{\sqrt{2 \pi \sigma_{E}^{2}(b)}} \exp \left(-\frac{\left[E_{T}-\bar{E}_{T}(b)\right]^{2}}{2 \sigma_{E}^{2}(b)}\right), .
$$

The total $E_{T}$ distribution is a convolution of the hard and soft components with mean and standard deviation

$$
\bar{E}_{T}(b)=\sum_{f} \bar{E}_{T}^{f}(b)+T_{A B}(b) \epsilon_{0} \quad \sigma_{E}^{2}(b)=\sum_{f} \overline{E_{T}^{2 f}}-\frac{\bar{E}_{T}^{H 2}}{\sigma^{H}\left(p_{0}\right)}+T_{A B}(b)\left(\epsilon_{1}-\frac{\epsilon_{0}^{2}}{\sigma_{p p}^{S}}\right) .
$$

The $E_{T}$ distributions are shown for CMS and STAR in Fig. 2. At the LHC, 90\% of the average $E_{T}$ comes from the hard component, causing the maximum $E_{T}$ to be halved due to shadowing. In fact, the low $x$ gluon density is high enough for the incoming gluon to have multiple hard collisions, up to 5 in central collisions with no shadowing, as it traverses the nucleus when the GRV 94 LO distributions are used. In contrast, an incoming quark has at most 1 collision. Similar results with MRST LO distributions typically halve the hard $E_{T}$ and significantly reduce gluon multiple scattering. Gluons suffer 1.4-2 hard collisions 
while quarks scatter less than once [12]. At RHIC, since the hard and soft components are comparable, the maximum $E_{T}$ is shifted by only $\sim 7 \%$ when shadowing is included. Indeed, for $S_{3}$, the maximum $E_{T}$ is slightly increased. Thus shadowing significantly affects the initial conditions at LHC but not at RHIC. Ref. [12] gives more details and discusses inhomogeneous shadowing effects on $J / \psi$ and Drell-Yan production.

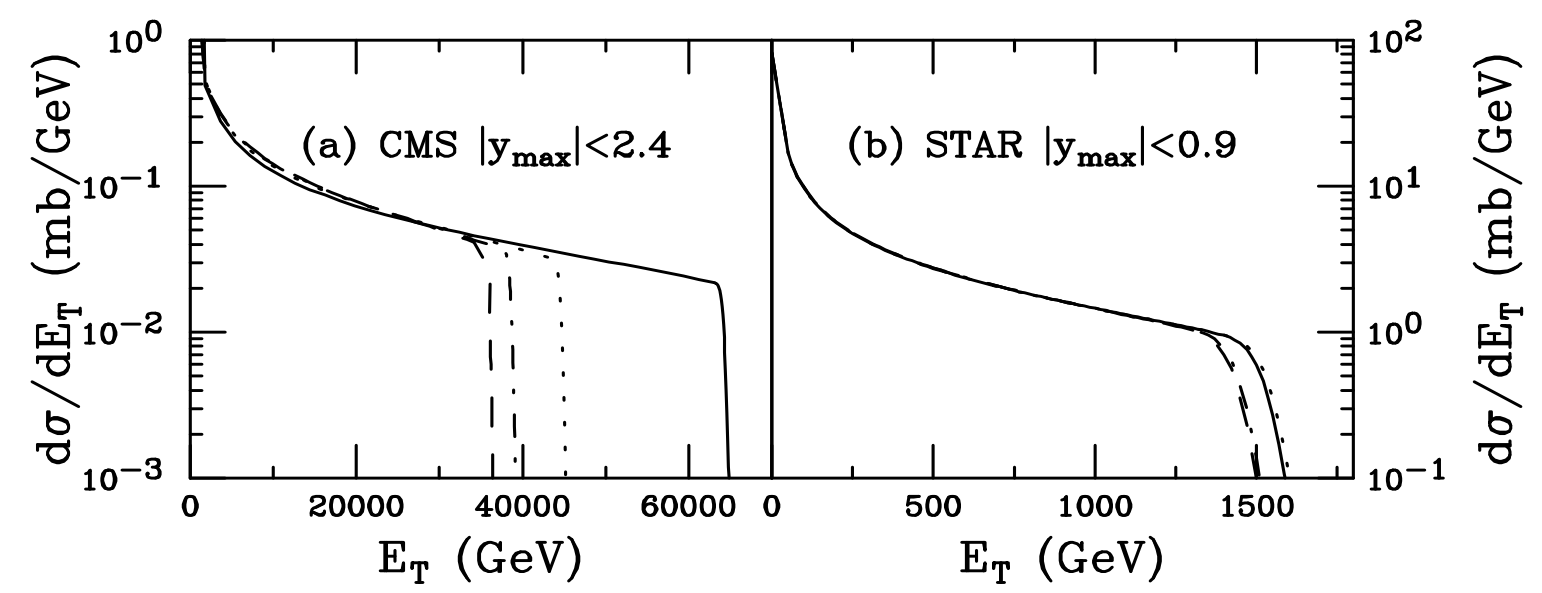

Figure 2. The $E_{T}$ distribution predicted for CMS, $\left|y_{\max }\right| \leq 2.4$, at the LHC and STAR, $\left|y_{\text {max }}\right| \leq 0.9$, at RHIC calculated with the GRV 94 LO distributions and $p_{0}=2 \mathrm{GeV}$. The homogeneous shadowing results for all $b$ are: no shadowing (solid), $S_{1}$ (dashed), $S_{2}$ (dot-dashed), and $S_{3}$ (dotted). Modified from Ref. [12].

\section{REFERENCES}

1. J.J. Aubert et al., Nucl. Phys. B293 740, (1987); M. Arneodo, Phys. Rep. 240301 , (1994).

2. T. Kitagaki et al., Phys. Lett. 214, 281 (1988).

3. V. Emel'yanov, A. Khodinov, S.R. Klein and R. Vogt, Phys. Rev. C56, 2726 (1997).

4. K.J. Eskola and M. Gyulassy, Phys. Rev. C47 2329, (1993).

5. K.J. Eskola and K. Kajantie, Z. Phys. C75 515, (1997);

6. M. Glück, E. Reya, and A. Vogt, Z. Phys. C67 433, (1995).

7. A.D. Martin, R.G. Roberts, and W.J. Stirling, and R.S. Thorne, Eur. Phys. J. C4 (1998) 463; Phys. Lett. B443 (1998) 301.

8. K.J. Eskola, J. Qiu, and J. Czyzewski, private communication.

9. K.J. Eskola, Nucl. Phys. B400 240, (1993).

10. K.J. Eskola, these proceedings.

11. V. Emel'yanov, A. Khodinov, S.R. Klein and R. Vogt, Phys. Rev. Lett. 81, 1801 (1998).

12. V. Emel'yanov, A. Khodinov, S.R. Klein and R. Vogt, LBNL-42900. 